\title{
Dynamic Magneto-mechanical Behavior of Magnetization-graded Ferromagnetic Materials
}

\author{
Lei Chen ${ }^{1,2 *}$ and Yao Wang ${ }^{3}$ \\ ${ }^{1}$ Key Lab of Computer Vision and Intelligent Information System, Chongqing University of Arts and Sciences, \\ Chongqing 402160, China \\ ${ }^{2}$ College of Optoelectronic Engineering, Chongqing University, Chongqing City, 400044, P.R. China \\ ${ }^{3}$ Electrical and Computer Engineering Department, University of Minnesota, Minneapolis, MN 55455, USA
}

(Received 7 April 2014, Received in final form 11 June 2014, Accepted 18 June 2014)

\begin{abstract}
This study investigates the dynamic magneto-mechanical behavior of magnetization-graded ferromagnetic materials Terfenol-D/FeCuNbSiB (MF). We measure the dynamic magneto-mechanical properties as a function of the DC bias magnetic field $\left(H_{\mathrm{dc}}\right)$. Our experimental results show that these dynamic magneto-mechanical properties are strongly dependent on the DC bias magnetic field. Furthermore, the dynamic strain coefficient, electromechanical resonance frequency, Young's moduli, and mechanical quality factor of Terfenol-D/FeCuNbSiB are greater than those of Terfenol-D under a lower DC bias magnetic field. The dynamic strain coefficient increases by a factor of between one and three, under the same DC bias magnetic field. In particular, the dynamic strain coefficient of Terfenol-D/FeCuNbSiB at zero bias achieves $48.6 \mathrm{~nm} / \mathrm{A}$, which is about 3.05 times larger than that of Terfenol-D. These good performances indicate that magnetization-graded ferromagnetic materials show promise for application in magnetic sensors.
\end{abstract}

Keywords : dynamic magnetomechanical properties, dynamic strain coefficient, magnetization-graded ferromagnetic materials, magnetic sensor, magnetoelectric effect

\section{Introduction}

A ferromagnetic material develops large mechanical deformations, and causes them to change their shape when subjected to an external magnetic field. This phenomenon is called magnetostriction [1]. The cause of magnetostrictional change in shape is the result of the rotation of small magnetic domains in the material, which are randomly oriented when the material is not exposed to a magnetic field. The orientation of these small domains by the imposition of a magnetic field creates a strain field, which leads to the stretching or shrinking of the material in the direction of the magnetization, as a function of the applied magnetic field. Typical ferromagnetic materials are Terfenol-D, Nickel, $\mathrm{FeNi}$, Metglas, $\mathrm{FeGa}$ and $\mathrm{SmFe}$ alloys, which have been successfully used in transducer, sonar, and actuator devices [2-5]. In particular, Terfenol-D $\left(\mathrm{Tb}_{\mathrm{x}} \mathrm{Dy}_{1-\mathrm{x}} \mathrm{Fe}_{\mathrm{y}}\right)$ has received significant attention over the past decades, due to its giant magnetostrictive strain (1200 ppm), strain energy density

(C)The Korean Magnetics Society. All rights reserved.

*Corresponding author: Tel: +86-23-49891553

Fax:+86-23-49891553, e-mail: 67638515@163.com $\left(20 \mathrm{~kJ} / \mathrm{m}^{3}\right)$, and good magneto-mechanical coupling property $[3,6]$. However, its small mechanical quality factor (ranging from 3 to 20), and quite low relative permeability (ranging from 3 to 10) lead to a low effective static strain coefficient (piezomagnetic coefficient) and dynamic strain coefficient (resonant strain coefficient) for Terfenol-D under a low $H_{\mathrm{dc}}$, and to larger required magnetic biases. This causes difficulties in machining and device fabrication, and limits practical applications. In response to these shortcomings, Dong et al. have reported that a higher effective piezomagnetic coefficient can be achieved by incorporating a high permeability $\mathrm{MnZnFe}_{2} \mathrm{O}_{4}$ layer into Terfenol-D, due to magnetic flux concentration [7]. Unfortunately, this will greatly increase the size, which is disadvantageous to practical applications. Recently, Srinivasan et al. [8] have proposed an internal magnetic field induced by a spatially varying magnetization in a compositionally graded ferromagnetic material. Taking advantage of this, we prepared magnetization-graded ferromagnetic materials Terfenol-D/ $\mathrm{FeCuNbSiB}$ (MF), consisting of the two different ferromagnetic materials, Terfenol-D and $\mathrm{FeCuNbSiB}\left(\mathrm{Fe}_{73.5} \mathrm{Cu}_{1} \mathrm{Nb}_{3^{-}}\right.$ $\left.\mathrm{Si}_{13.5} \mathrm{~B}_{9}\right)$. Due to their different magnetic characteristics, an internal magnetic field $\left(H_{\text {int }}\right)$ is produced. Accordingly, the 
total DC magnetic bias $\left(H_{\text {total,dc }}\right)$ consists of an external DC magnetic field $\left(H_{\mathrm{dc}}\right)$ and $H_{\mathrm{int}}$, which results in large strain coefficients under a low $H_{\mathrm{dc}}$, due to the strain coefficient dependence on the magnetic field. The benefits of using these magnetization-graded ferromagnetic materials are that they increase the strain coefficient under a low field, and maintain its small size.

The behavior of the magnetization-graded ferromagnetic materials in various applications is complex, because the changing conditions during operation cause changes in the material properties. A full understanding of the complexity will enable engineers to use the potential advantages of magnetization-graded ferromagnetic materials. In this paper, we discuss the dynamic behavior of magnetization-graded ferromagnetic materials Terfenol-D/FeCuNbSiB. We investigate the dependence of the dynamic magneto-mechanical properties on the DC bias magnetic field and frequency. We evaluate the dynamic performance by measuring its vibrational characteristics with a laser doppler vibrometer (LDV) [4]. The experimental results show that MF in a low magnetic field can obtain a relatively high dynamic strain coefficient, due to the internal magnetic field, compared with Terfenol-D. In particular, the dynamic strain coefficient for MF can achieve $48.5 \mathrm{~nm} / \mathrm{A}$ at zero bias. In addition, the dynamic strain coefficient and the mechanical quality factor for MF strongly depend on the DC bias magnetic field. The good performance indicated that Terfenol$\mathrm{D} / \mathrm{FeCuNbSiB}$ would be a promising ferromagnetic material for magnetic sensor applications.

\section{Experiment}

Samples of the magnetization-graded ferromagnetic materials Terfenol-D/FeCuNbSiB, employing Fe-based nanocrystalline $\mathrm{FeCuNbSiB}$ alloy (International standard trademark 1K107, provided by Foshan Huaxin Microlite Metal Co., Ltd., China) and Terfenol-D (Taizhou Jiaoguang Rare Earth Materials Co., Ltd., China), were prepared, as shown in Fig. 1. The Terfenol-D plate was cut into rectangular plates of dimensions $12 \mathrm{~mm} \times 6 \mathrm{~mm} \times 1 \mathrm{~mm}$. The $\mathrm{FeCuNbSiB}$ ribbon is a material of high magnetic permeability $\left(\mu_{\mathrm{r}}>\right.$ $10000)$, high saturation magnetization $\left(\mu_{0} \mathrm{M}_{\mathrm{s}}=1.45 \mathrm{~T}\right)$, low magnetostriction $(\lambda=2.7 \mathrm{ppm})$, and large anisotropic constant $\left(-30000 \mathrm{~J} / \mathrm{m}^{3}\right)$ [9]. Its dimensions are $12 \mathrm{~mm} \times 6 \mathrm{~mm}$ $\times 0.03 \mathrm{~mm}$. The $\mathrm{FeCuNbSiB}$ layer was prepared by the stacking and bonding of two $\mathrm{FeCuNbSiB}$ ribbons. The magnetization-graded ferromagnetic materials Terfenol-D/ $\mathrm{FeCuNbSiB}$ was bonded using an epoxy adhesive, and cured at $80{ }^{\circ} \mathrm{C}$ for $1 \mathrm{~h}$ under load, to provide a strong bond between the layers. The two ferromagnetic materials were both magnetized along the longitudinal direction.

The experimental setup for investigating the dynamic magneto-mechanical behavior is shown in Fig. 2. A pair of annular permanent magnets $(\mathrm{NdFeB})$ was used to generate

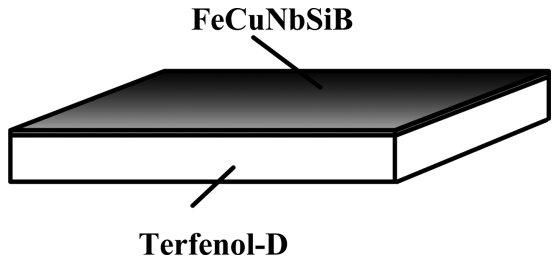

Fig. 1. Schematics of the magnetization-graded ferromagnetic materials Terfenol-D/FeCuNbSiB.

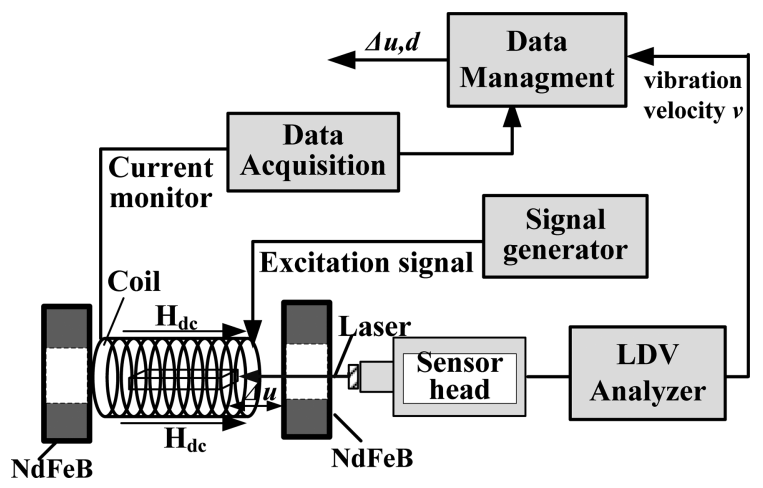

Fig. 2. Schematic diagram of the dynamic measurement setup.

the DC bias magnetic field $\left(H_{\mathrm{dc}}\right)$, which was monitored by Gauss meter. A constant current was applied in a helix coil with a turn number of 245 , cylindrical length of $67 \mathrm{~mm}$, and cylindrical diameter of $41 \mathrm{~mm}$; correspondingly, an $\mathrm{AC}$ magnetic field $\left(H_{\mathrm{ac}}\right)$ was excited at the center of the cylindrical shell. The measured sample was placed at the center of the solenoid, in which AC and DC uniform magnetic fields were generated along the longitudinal direction. It was driven to vibrate around the $\lambda / 2$ longitudinal resonance, by $H_{\mathrm{ac}}$ superimposed $H_{\mathrm{dc}}$. The vibration velocity $v$ at the end faces of the samples was measured by Laser Doppler vibrometer (Polytec Model OFV-5000, Germany) [4]. The dynamic strain coefficient of the sample was calculated by the equation $d_{33, \mathrm{~m}}=d \lambda / d \mathrm{H}=v /\left(\pi f l H_{\mathrm{ac}}\right)$, where $v$ is the vibration velocity of the sample induced by the magnetic field, $f$ is the exciting frequency, $l$ is the undeformed length of sample, and $H_{\text {ac }}$ is the magnitude of the external AC magnetic field [4]. The experiments were carried out at room temperature and ambient pressure.

\section{Results and Discussion}

Fig. 3 shows the measured dynamic strain coefficient as a function of frequency (strain coefficient spectra) for the magnetization-graded ferromagnetic materials MF near resonance, under various magnetic fields $H_{\mathrm{dc}}$.

The figure shows the fundamental longitudinal mode resonance of the sample to be approximately $100 \mathrm{kHz}$. A sharp resonance peak is observed for $H_{\mathrm{dc}}=19,55,105$, 235,598 , and 700Oe. A shift occurs in the resonant peak, 


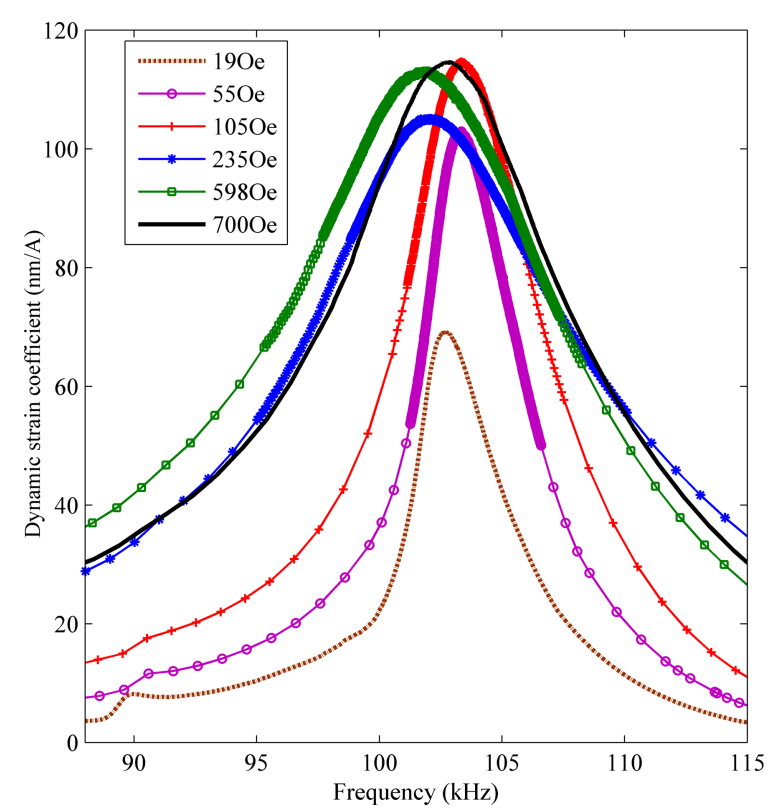

Fig. 3. (Color online) Variations of the dynamic strain coefficient for the magnetization-graded ferromagnetic materials Terfenol-D/FeCuNbSiB with resonant frequency $f_{\mathrm{r}}$, at several different values of $H_{\mathrm{dc}}$ ranging from 19 Oe to 700 Oe.

with the DC bias magnetic field increasing from 19 Oe to 700 Oe. As can be seen, the maximum dynamic strain coefficient achieves $114.8 \mathrm{~nm} / \mathrm{A}$ at the resonance frequency of $\sim 102.86 \mathrm{kHz}$ under $H_{\mathrm{dc}}=105 \mathrm{Oe}$, which is much larger than its non-resonance values.

As shown in Fig. 4 the dynamic strain coefficients for the Terfenol-D and Terfenol-D/FeCuNbSiB exhibit significant dependence on the DC magnetic bias. The dependence of

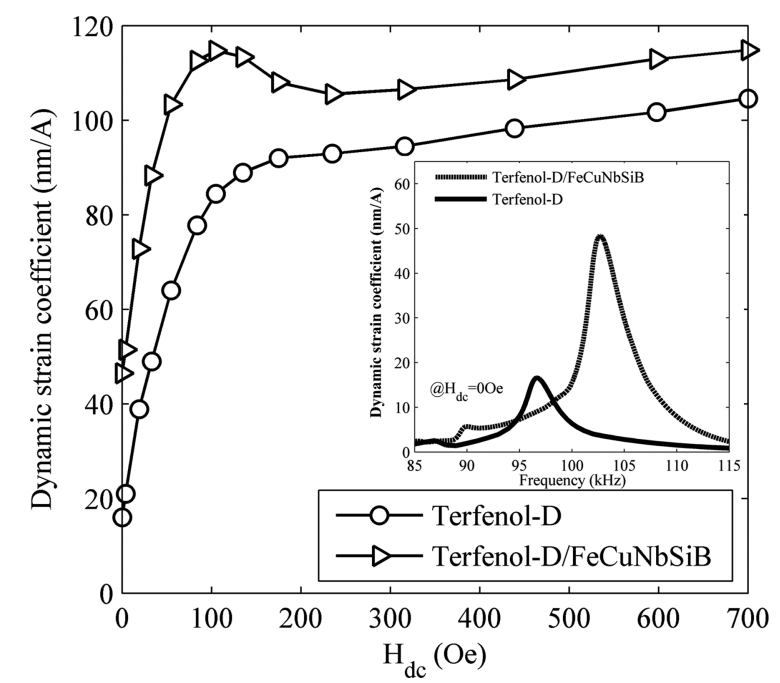

Fig. 4. The dynamic strain coefficient for Terfenol-D and the magnetization-graded ferromagnetic materials Terfenol-D/ $\mathrm{FeCuNbSiB}$ as a function of $H_{\mathrm{dc}}$. The inset shows the dynamic strain coefficient as a function of frequency, around the electromechanical resonance frequency at $H_{\mathrm{dc}}=0 \mathrm{Oe}$. the dynamic strain coefficient on $H_{\mathrm{dc}}$ originates from the piezomagnetic coefficient $d_{33}$ dependence on $H_{\mathrm{dc}}$. Generally, the piezomagnetic coefficient for magnetostrictive material increases, and achieves a certain maximum; then decreases slowly, with increasing applied DC bias magnetic field. From Fig. 4, we can see that the dynamic strain coefficient for Terfenol-D increases up to its maximum of $104.6 \mathrm{~nm} / \mathrm{A}$, with DC bias magnetic field increasing up to $700 \mathrm{Oe}$. In comparison, we find that the dynamic strain coefficient for the magnetization-graded ferromagnetic materials Terfenol$\mathrm{D} / \mathrm{FeCuNbSiB}$ increases dramatically up to a maximum value $(114.8 \mathrm{~nm} / \mathrm{A})$ near $105 \mathrm{Oe}$, and then slowly decreases until $H_{\mathrm{dc}}=235 \mathrm{Oe}$; and next, slightly increases, with increasing DC bias field. Under lower DC magnetic field biases of $H_{\mathrm{dc}}<105 \mathrm{Oe}$, the dynamic strain coefficient for Terfenol$\mathrm{D} / \mathrm{FeCuNbSiB}$ is increased by a factor of between one and three under the same DC magnetic field bias, compared with Terfenol-D. In particular, the effective dynamic strain coefficient of Terfenol-D/FeCuNbSiB under $H_{\mathrm{dc}}=0$ Oe achieves $48.6 \mathrm{~nm} / \mathrm{A}$, due to the internal magnetic field induced by the spatially varying magnetization, which is about 3.05 times larger than that of Terfenol-D (as shown in the inset of Fig. 4). There are some significant differences between the trends of the dynamic strain coefficient for Terfenol-D and Terfenol-D/FeCuNbSiB laminated composite with $H_{\mathrm{dc}}$. This is principally because the dynamic strain coefficient is directly proportional to the product of the effective mechanical quality factor $Q_{\mathrm{m}}$ and the piezomagnetic coefficient $d_{33}$ [10]. The mechanical quality factor $Q_{\mathrm{m}}$ strongly depends on $H_{\mathrm{dc}}$ (as shown in Fig. 5). On the basis of the dynamic strain coefficient spectrum under various $H_{\mathrm{dc}}$, the effective mechanical quality factor of the Terfenol$\mathrm{D}$ and Terfenol-D/FeCuNbSiB is calculated by:

$$
Q_{m}=\frac{f_{r}}{\Delta f}
$$

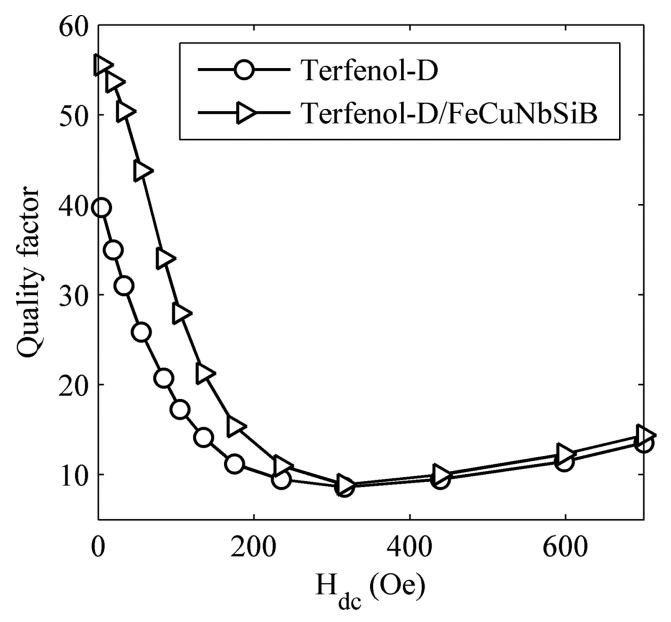

Fig. 5. Mechanical quality factors for Terfenol-D and the magnetization-graded ferromagnetic materials Terfenol-D/ FeCuNbSiB composite, as a function of $H_{\mathrm{dc}}$. 
where, $f_{\mathrm{r}}$ is the resonance frequency, and $\Delta f$ is the $3-\mathrm{dB}$ bandwidth.

In comparison to the variation of the piezomagnetic coefficient, as $H_{\mathrm{dc}}$ increases, $Q_{\mathrm{m}}$ first rapidly decreases, until it reaches a certain minimum; and then it gradually increases (as shown in Fig. 5). We can see that the effective $Q_{\mathrm{m}}$ of the Terfenol-D/FeCuNbSiB is greater than that of Terfenol-D, under the lower DC magnetic field biases of $H_{\mathrm{dc}}<105 \mathrm{Oe}$, because $Q_{\mathrm{m}}$ is a measure of mechanical losses or damping in a material, with inversely proportional relationship. These losses can be regarded as mechanical hysteresis losses, which generally limit the dynamic behavior of the material. Obviously, a larger mechanical quality factor $Q_{\mathrm{m}}$ for Terfenol-D/FeCuNbSiB can be obtained, owing to the high $Q_{\mathrm{m}}$ of FeCuNbSiB (of about 1000), resulting in the higher product of the effective mechanical quality factor $Q_{\mathrm{m}}$ and the piezomagnetic coefficient. Correspondingly, the dynamic strain coefficient for the magnetization-graded ferromagnetic materials Terfenol- $\mathrm{D} / \mathrm{FeCuNbSiB}$ increases more rapidly under $H_{\mathrm{dc}}<105 \mathrm{Oe}$, relative to Terfenol-D.

Fig. 6 shows the DC bias magnetic field $H_{\mathrm{dc}}$ dependence of the resonance frequency $f_{\mathrm{r}}$ for Terfenol-D and Terfenol$\mathrm{D} / \mathrm{FeCuNbSiB}$. The resonance frequency depends on $H_{\mathrm{dc}}$, which can be explained by the $\Delta \mathrm{E}$ effect and motion of the non- $180^{\circ}$ domain walls. For the Terfenol-D, the resonant frequency $f_{\mathrm{r}}$ reduces quickly to a minimum value at $H_{\mathrm{dc}}=$ $316 \mathrm{Oe}$, and then slightly increases with the DC bias magnetic field, showing a "V" shape in the range of 0-700Oe (as shown in Fig. 6(a)). Furthermore, it demonstrates a pronounced shifting of the resonance frequency, from $f_{\mathrm{r}}=$ $92.93 \mathrm{kHz}$ for $H_{\mathrm{dc}}=316 \mathrm{Oe}$, to $f_{\mathrm{r}}=98.69 \mathrm{kHz}$ for $H_{\mathrm{dc}}=$ 700 Oe. In contrast, as $H_{\mathrm{dc}}$ increases, the resonant frequency for the magnetization-graded ferromagnetic materials Terfenol- $\mathrm{D} / \mathrm{FeCuNbSiB}$ first increases, until it reaches a maximum value at $H_{\mathrm{dc}}=84 \mathrm{Oe}$; and then it decreases quickly, to a minimum value at $H_{\mathrm{dc}}=439 \mathrm{Oe}$; and next, it slowly increases, as the field increases (as shown in Fig. 6(b)). The resonance frequency shifts over the range of $99.54 \mathrm{kHz}-$
$103.4 \mathrm{kHz}$, with increasing $H_{\mathrm{dc}}$ from 0 Oe to $700 \mathrm{Oe}$. Some discrepancies are induced by the variation of Young's modulus of the $\mathrm{FeCuNbSiB}$ material with the applied DC magnetic field. The mechanical resonance frequency for the magnetization-graded ferromagnetic materials Terfenol-D/ $\mathrm{FeCuNbSiB}$ can be written as:

$$
f_{r}=\frac{1}{2 l} \sqrt{\frac{\bar{E}}{\bar{\rho}}}
$$

where, $\bar{\rho}$ is the average density of the composite determined by $\bar{\rho}=n_{f} \rho_{f}+\left(1-n_{f}\right) \rho_{t}$, and $\rho_{f}$ and $\rho_{t}$ are the Young's moduli of FeCuNbSiB and Terfenol-D, respectively. The equivalent Young's modulus $\bar{E}$ is given by

$$
\bar{E}=n_{f} E_{f}+\left(1-n_{f}\right) E_{t}
$$

where, $\mathrm{E}_{f}$ and $\mathrm{E}_{m}$ are the Young's moduli of $\mathrm{FeCuNbSiB}$ and Terfenol-D, respectively, and $\mathrm{n}_{f}$ is the volume fraction of the $\mathrm{FeCuNbSiB}$ layer.

The equivalent Young's modulus for the magnetizationgraded ferromagnetic materials changes, compared with Terfenol-D (as shown in Figs. 7(a) and (b)). As a result, the resonance frequency for Terfenol-D/FeCuNbSiB changes along with the Young's modulus. Furthermore, the relatively high Young's modulus of $\mathrm{FeCuNbSiB}$ enhances the equivalent Young's modulus, and the relatively small density of $\mathrm{FeCuNbSiB}$ reduces the average density according to Eqs. (2) and (3). Consequently, the resonance frequency of the magnetization-graded ferromagnetic materials Terfenol-D/ $\mathrm{FeCuNbSiB}$ is higher than that of Terfenol-D.

The magnetization-graded ferromagnetic materials Terfenol$\mathrm{D} / \mathrm{FeCuNbSiB}$ is a potentially feasible material for use in high-sensitivity $\mathrm{DC}$ or/and $\mathrm{AC}$ magnetic field detection. One approach would be to prepare a PZT/Terfenol-D/ $\mathrm{FeCuNbSiB}$ magnetoelectric (ME) laminated composite, by combining a piezoelectric material PZT with Terfenol-D/ $\mathrm{FeCuNbSiB}$. Since the dynamic strain coefficient of the magnetization-graded ferromagnetic materials is intrinsical-
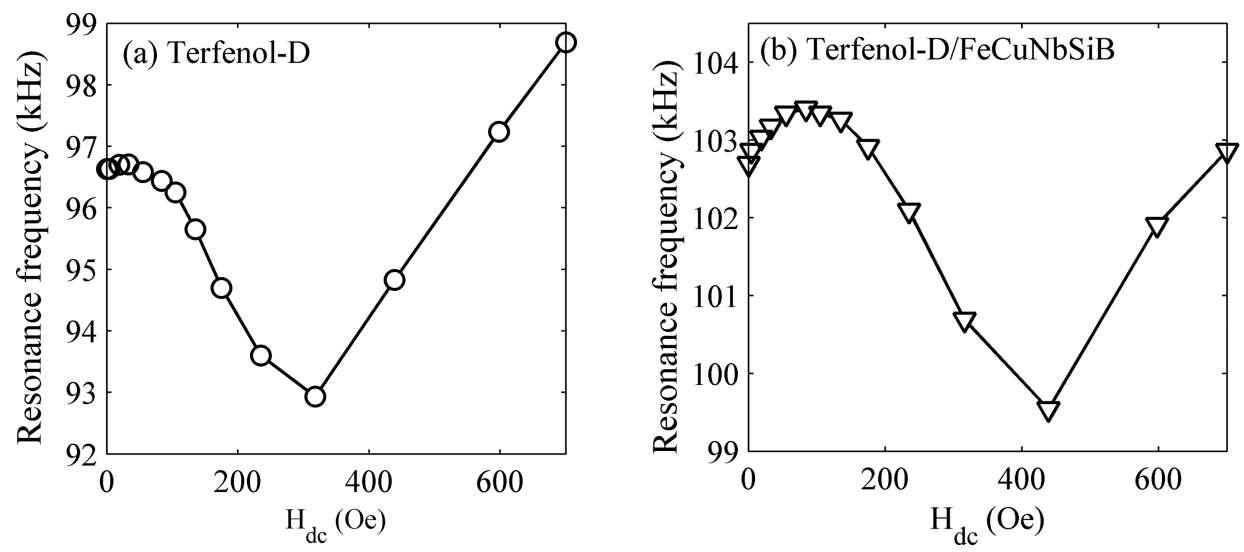

Fig. 6. Variations of resonant frequencies for Terfenol-D and the magnetization-graded ferromagnetic materials Terfenol-D/ $\mathrm{FeCuNbSiB}$, with $H_{\mathrm{dc}}$ ranging from 0 Oe to $700 \mathrm{Oe}$. 

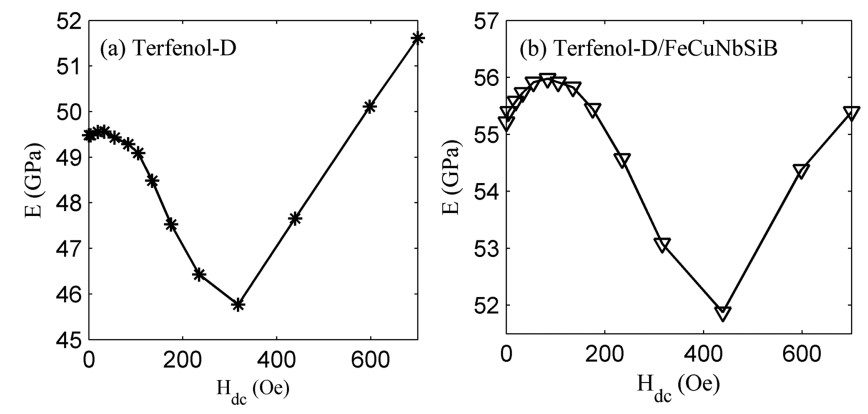

Fig. 7. The Young's moduli for Terfenol-D and the magnetization-graded ferromagnetic materials Terfenol-D/FeCuNbSiB, as a function of the DC bias magnetic field.

ly dependent on the DC bias magnetic field $H_{\mathrm{dc}}$, such a sensor can detect a small $H_{\mathrm{dc}}$ signal, by using the operational mode proposed in Ref. [11]. In the ME measurement, the Terfenol-D/FeCuNbSiB (the easy direction is along the longitudinal direction) is magnetized in the longitudinal (length) direction, and the PZT is polarized in the transverse (thickness) direction. Fig. 8 shows the induced ME voltage for $\mathrm{PZT} / \mathrm{Terfenol}-\mathrm{D} / \mathrm{FeCuNbSiB}$, and the phase shift as a function of the dc magnetic field, when an ac magnetic field $\left(H_{\mathrm{ac}}\right)$ with a peak-peak value of 1 Oe and a resonance frequency of $36 \mathrm{kHz}$ is applied. The measured ME voltage shows an unexpected $180^{\circ}$ phase shift, upon reversal of the sign of $H_{\mathrm{dc}}$. Such reversals are found under small dc magnetic fields of $H_{\mathrm{dc}}= \pm 67$ Oe. The phase shift reveals the tendency of the applied dc field variation [11]. According to Fig. 8, the maximum ME sensitivity of the ME voltage $V_{\mathrm{ME}}$ to $H_{\mathrm{dc}}$ (i.e., $\mathrm{d} V_{\mathrm{ME}} / \mathrm{d} H_{\mathrm{dc}}$ under $\mathrm{AC}$ bias field) reaches $125.9 \mathrm{mV} / \mathrm{Oe}$ under a resonant drive of $H_{\mathrm{ac}}=1 \mathrm{Oe}$, which is about 5 times higher than that of PZT/Terfenol-D sensor $(25.7 \mathrm{mV} / \mathrm{Oe})$. The higher sensitivity of the ME

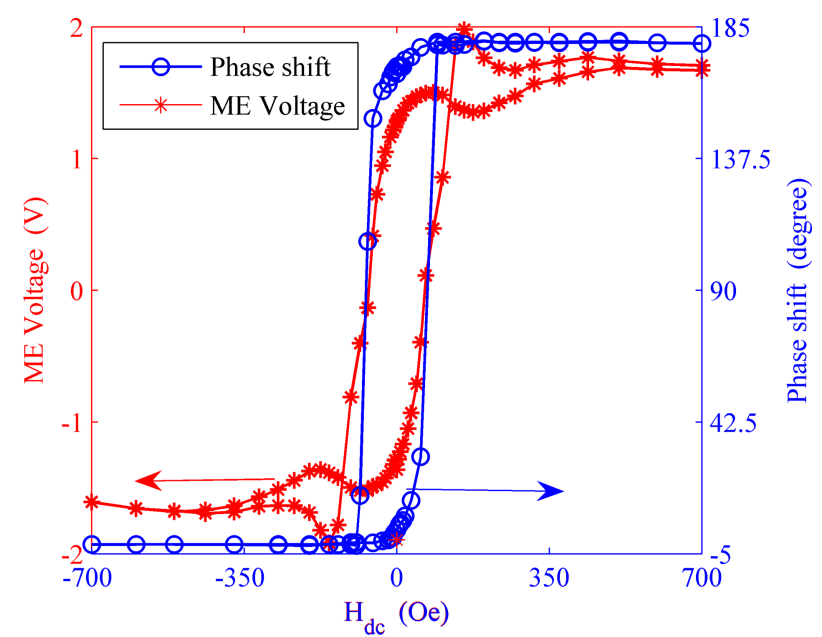

Fig. 8. (Color online) The ME voltage and phase shift as a function of the DC magnetic field bias for ME sensor, under a resonant drive of $H_{\mathrm{ac}}=1$ Oe. sensor is mainly due to a higher dynamic strain coefficient for Terfenol- $\mathrm{D} / \mathrm{FeCuNbSiB}$, which results in a higher output voltage in response to a small variation of $H_{\mathrm{dc}}$. Furthermore, we can clearly see that the zero-biased ME voltage of the ME composite has a giant value of $1.362 \mathrm{~V}$. Correspondingly, the ME sensitivity of the ME voltage $V_{\mathrm{ME}}$ to $H_{\mathrm{ac}}$ (i.e., $\mathrm{d} V_{\mathrm{ME}} / \mathrm{d} H_{\mathrm{ac}}$ under a DC bias field) is calculated as $1.362 \mathrm{~V} /$ Oe $(17.025(\mathrm{~V} / \mathrm{cm} \mathrm{Oe}))$. This indicates the possibility of using the PZT/Terfenol-D/FeCuNbSiB ME sensor for lowlevel ac magnetic field detection without bias, which can greatly reduce the size, relative to the traditional ME sensor. Obviously, this ME sensor can sense not only an $\mathrm{AC}$ magnetic field, but also, and in particular, a DC magnetic field.

\section{Conclusion}

We investigate the dynamic magneto-mechanical behavior of magnetization-graded ferromagnetic materials Terfenol$\mathrm{D} / \mathrm{FeCuNbSiB}$ (MF) relative to a DC bias magnetic field. The dynamic strain coefficient, mechanical resonance frequency, Young's modulus, and mechanical quality factor are strongly dependent on the DC bias magnetic field. The MF displays a significantly high dynamic strain coefficient, and a large effective mechanical quality factor, compared with Terfenol-D. In particular, the dynamic strain coefficient for MF can achieve $48.5 \mathrm{~nm} / \mathrm{A}$ at zero bias. Based on dynamic magneto-mechanical characteristics, we fabricate a new type of sensor with PZT/Terfenol-D/FeCuNbSiB. The maximum ME sensitivity of the ME voltage $V_{\mathrm{ME}}$ to $H_{\mathrm{dc}}$ reaches $125.9 \mathrm{mV} / \mathrm{Oe}$ under a resonant drive of $H_{\mathrm{ac}}=1 \mathrm{Oe}$, which about 5 times higher than that of PZT/Terfenol-D sensor $(25.7 \mathrm{mV} / \mathrm{Oe})$. The ME sensitivity of the ME voltage $V_{\mathrm{ME}}$ to $H_{\mathrm{ac}}$ achieves $1.362 \mathrm{~V} / \mathrm{Oe}(17.025(\mathrm{~V} / \mathrm{cm} \mathrm{Oe}))$ at zero bias. These properties are promising for use in magnetic field sensing.

\section{Acknowledgment}

This research is supported by the National Natural Science Foundation of China (Grant No. 61304255), the China Postdoctoral Science Foundation (No. 2013M530393 and No. 2014T70851), the Scientific and Technological Research Program of Chongqing Municipal Education Commission (No. KJ131221), the Chongqing Postdoctoral Science Special Foundation (No. Xm201304).

\section{References}

[1] G. Engdahl, Magnetostrictive Materials Handbook, San Diego: Academic Press (2000).

[2] W. Huang, B. Wang, Y. Sun, L. Weng, and W. Zhao, Proc. SPIE 6423, 64234I (2007).

[3] S. W. Or, N. Nersessian, and G. P. Carman, Proc. SPIE 
4699, 451 (2002).

[4] L. X. Bian, Y. M. Wen, P. Li, Q. L. Gao, and X. X. Liu, J. Magn. 14, 66 (2009).

[5] L. Chen, P. Li, and Y. M. Wen, J. Magn. 16, 3 (2011).

[6] S. W. Or, N. Nersessian, and G. P. Carman, J. Magn. Magn. Mater. 262, 181 (2003).

[7] S. Dong, J. Zhai, J. F. Li, and D. Viehland, J. Appl. Phys. 100, 124108 (2006).

[8] C. Sudakar, R. Naik, G. Lawes, J. V. Mantese, A. L.
Micheli, G. Srinivasan, and S. P. Alpay, Appl. Phys. Lett. 90, 062502 (2007).

[9] S. Hong, J. G. Kim, and C. G. Kim, J. Magn. 14, 71 (2009).

[10] F. Claeyssen, N. Lhermet, R. Le Letty, and P. Bouchilloux, J. Alloys Compd. 258, 61 (1997).

[11] S. X. Dong, J. Y. Zhai, J. F. Li, and D. Viehland, Appl. Phys. Lett. 88, 082907 (2006). 